Submitted to Hearing Research, June 2015

\title{
Binaural beats increase interhemispheric alpha-band coherence between auditory cortices
}

\author{
Marco Solcà $^{1}$, Anaïs Mottaz ${ }^{1}$, Adrian G. Guggisberg ${ }^{1}$ \\ 1 Division of Neurorehabilitation, Department of Clinical Neurosciences, University \\ Hospital and University of Geneva, CH-1211 Geneva 14, Switzerland
}

Running title: EEG Coherence \& Binaural Beats

\section{Correspondance:}

Adrian G. Guggisberg

Service de Neurorééducation

Hôpitaux Universitaires de Genève

Av. de Beau-Séjour 26

CH-1211 Geneva 14 / Switzerland

Tel.: +41-22-382 3620; Fax: +41-22-382 3644

e-mail: adrian.guggisberg@hcuge.ch 


\section{Abstract}

Binaural beats (BBs) are an auditory illusion occurring when two tones of slightly different frequency are presented separately to each ear. BBs have been suggested to alter physiological and cognitive processes through synchronization of the brain hemispheres. To test this, we recorded electroencephalograms (EEG) at rest and while participants listened to BBs or a monaural control condition during which both tones were presented to both ears. We calculated for each condition the interhemispheric coherence, which expressed the synchrony between neural oscillations of both hemispheres. Compared to monaural beats and resting state, BBs enhanced interhemispheric coherence between the auditory cortices. Beat frequencies in the alpha $(10 \mathrm{~Hz})$ and theta $(4 \mathrm{~Hz})$ frequency range both increased interhemispheric coherence selectively at alpha frequencies. In a second experiment, we evaluated whether this coherence increase has a behavioral aftereffect on binaural listening. No effects were observed in a dichotic digit task performed immediately after BBs presentation. Our results suggest that BBs enhance alpha-band oscillation synchrony between the auditory cortices during auditory stimulation. This effect seems to reflect binaural integration rather than entrainment.

Keywords: Functional connectivity, alpha band, EEG, phase synchrony, dichotic digit test, Interhemispheric synchronization 


\section{Introduction}

Human brain function crucially depends on interregional neural communication (Varela, et al., 2001). Such interactions are thought to be accompanied by a synchronization of oscillations between different brain regions (Aertsen, et al., 1989; Gray \& Singer, 1989; Engel, et al., 1992; Fries, 2005). Interregional synchronization can be quantified with the concept of functional connectivity (FC), which is a measure of the statistical dependency between activities in different brain regions. Interestingly, FC has been shown to linearly correlate with behavioral performance in a variety of cognitive and motor functions. In other words, the more synchronous neural oscillations are between nodes of a given network, the better is the behavioral performance in tasks relying on that network (Hummel, et al., 2005; Fox, et al., 2007; Guggisberg, et al., 2015). Therefore, interventions leading to modulation of FC should have beneficial behavioral effects and could lead to new applications and therapies.

One intervention that has been suggested to provide such modulation of neural oscillation synchrony is the binaural presentation of tones with slightly different frequencies (Atwater, 2004). If two sinusoidal tones with slightly different frequencies are played simultaneously, one perceives a tone with their average frequency plus a so-called beat, i.e., a modulation of the amplitude at a rate equal to the difference between them. For instance, a tone of $395 \mathrm{~Hz}$ presented together with a tone of $405 \mathrm{~Hz}$ will produce a perceived frequency of $400 \mathrm{~Hz}$, which modulates in amplitude with a frequency of $10 \mathrm{~Hz}$ (Moore, 2012). If the two tones are presented separately to each ear, a similar beat is perceived by the subject although no such physical sound exists (Oster, 1973). This auditory illusion is known as BBs and has been interpreted as arising from the convergence of phase preserved neural activity from the two 
ears in binaurally sensitive neurons within the medial superior olivary nucleus (Wernick \& Starr, 1968; Kuwada, et al., 1979).

A common assumption of binaural beats is that they can elicit an entrainment effect of neural oscillation at their proper frequency (Vernon, 2009). Many electrophysiological studies have explored the effect of BBs on oscillatory amplitude with contradictory results. Some authors have reported increases in amplitude following exposure to binaural beats compared to resting state (Karino, 2004; Schwarz \& Taylor, 2005; Karino, et al., 2006; Draganova, et al., 2008; Pratt, et al., 2009; Pratt, et al., 2010; Grose \& Mamo, 2012) whilst others have failed to find evidence of such a change (Wahbeh, et al., 2007; Goodin, et al., 2012; D. Vernon, et al., 2014). However, it seems that when BBs increase power, it is always in smaller amplitude than the modulation occurring under the corresponding monaural beats (MBs) condition (Schwarz \& Taylor 2005; Draganova, et al., 2008; Pratt, et al., 2010).

It has further been argued that BBs enhance the synchrony of neural activity between the two brain hemispheres (Atwater, 1997; Atwater, 2004). Recently, two studies have tested the effect of BBs on FC. Gao and colleagues quantified frequency-specific synchronization between pairs of scalp electrodes (Gao, et al., 2014). They detected both increases and decreases in FC during BBs stimulation compared to a resting state without stimulation, depending on oscillation frequencies and electrode location. In another study, Becher and colleagues (Becher, et al., 2014) measured phase synchronization between channel pairs using intracranial signals mainly in the temporo-lateral and temporo-basal area. Synchronization increases were essentially found for stimulation with monaural $10-\mathrm{Hz}$ beats at mediotemporal sites and for stimulation with binaural 5-Hz beats at temporo-lateral sites. 
These two recent studies suggest that BBs could modulate oscillation synchrony. However, it remains unclear whether these changes occur in the BBs frequency, which brain structures are concerned and if they are associated with behavioral changes.

This last point is of particular interest because BBs have been praised for supposedly beneficial behavioral effects, such as relaxation, pain reduction, and other mental states (Wilson, 1990; Rhodes, 1993). Several studies have demonstrated specific behavioral changes following BBs such as improvement of vigilance (Lane, et al., 1998), increase in hypnotic susceptibility (Brady \& Stevens 2000), reduction of self-reported anxiety (Le Scouarnec, et al., 2001; Padmanabhan, et al., 2005), or increase in some forms of creativity (Reedijk, et al., 2013), although their robustness remains to be demonstrated. Given the assumed positive effect of BBs on synchronization between hemispheres, it would be particularly interesting to test whether such improved interhemipheric integration can be measured on the behavioral level. However, no study has probed the effect of BBs on tasks specifically requiring the collaboration between cerebral hemispheres in general or between both auditory cortices in particular.

The purpose of this study was therefore to directly test the hypothesis that BBs synchronize activity between hemispheres both at the neural and at the behavioral level. Experiment 1 examined the effect of BBs on interhemispheric oscillation synchrony in the auditory cortex using high-density scalp EEG and advanced source localization. Experiment 2 probed behavioral effects in a task requiring binaural listening. A dichotic listening task was used to assess the ability of participants to discriminate numbers presented simultaneously to different ears. This task has been validated for behavioral assessment of information exchange between the hemispheres across the corpus callosum (Musiek, 1983; Bellis \& Wilber, 2001; 
Westerhausen \& Hugdahl, 2008). We therefore used it to examine whether BBs improve the ability to exchange information between the auditory cortices even after stimulation.

\section{Materials and Methods}

The University Hospital of Geneva Ethics Committee approved all procedures and all subjects gave informed written consent for their participation to the study.

\subsection{Experiment 1}

\subsubsection{Data acquisition}

Sinusoidal tones of $395 \mathrm{~Hz}$ and $405 \mathrm{~Hz}$ (producing a beat of $10 \mathrm{~Hz}$ ), as well as $398 \mathrm{~Hz}$ and $402 \mathrm{~Hz}$ (producing a beat of $4 \mathrm{~Hz}$ ) were created using Matlab® (The MathWorks Inc., Natick, USA). These pairs of tones were presented with stereo headphones in two different conditions. In the monaural beats (MBs) condition, which was used as control, both tones were played to both ears (i.e. the beat is a purely physical phenomenon perceived by each ear). In the binaural beat condition, which was of interest here, each tone was played to separate ears (i.e. the beat is created by binaural integration and doesn't exist at the single ear level). Sound intensity was determined for each subject to be comfortably loud and kept constant between conditions.

Nine normal-hearing subjects (6 females, mean age of 28 years ranging from 25 to 34 years old) were asked to listen to $10 \mathrm{~Hz}$ BBs and $10 \mathrm{~Hz}$ MBs. Five participants were also exposed to $4 \mathrm{~Hz}$ BBs and the corresponding MBs. Each sound was presented continuously for 4 minutes in a counterbalanced order while a continuous EEG was recorded at a sampling rate of 1024 $\mathrm{Hz}$ using a 128-channel Biosemi Active Two EEG system (Biosemi B.V., Amsterdam, 
Netherlands). The participants were asked to listen to the sound. A resting state condition was also recorded before any auditory stimulation. All conditions were recorded with the eyes closed. Vigilance was checked by monitoring the EEG signal for signs of drowsiness or sleep and by asking the subjects whether they had fallen asleep after each condition. Artifacts like eye movements, muscular contractions and electrode artifacts were excluded by visual inspection. About three minutes of clean data were retained for each condition and each subject. Channels containing artifacts over prolonged periods were completely excluded from further analyses.

\subsubsection{Analysis}

Analyses were performed in Matlab with NUTMEG (http://nutmeg.berkeley.edu) (Dalal, et al., 2011) and its FCM toolbox (Guggisberg, et al., 2011).

We divided the three minutes artifact-free data into non-overlapping segments of one-second duration. Recordings were bandpass filtered between 1 and $20 \mathrm{~Hz}$. A spherical model with anatomical constraints (SMAC) (Spinelli, et al., 2000) was created from the individual coregistered magnetic resonance image of the head and used to compute the lead potential. The signal was then projected to grey matter voxels with an adaptive spatial filter (scalar minimum variance beamformer) (Sekihara, et al., 2004).

The Heschl Gyrus was defined as region of interest (ROI) using the Automated Anatomical Labeling (AAL) atlas (Tzourio-Mazoyer, et al., 2002) since we expected changes in the primary auditory cortex during auditory stimulation. 
Power spectrogram was computed using Fourier transformation. The difference in power between resting state, MBs and BBs conditions was compared in the Heschl Gyrus and tested against the null-hypothesis of zero change using two-tailed paired $t$-test.

The detailed steps of FC analysis and their validation have been described previously (Guggisberg, et al., 2008; Guggisberg, et al., 2011). We calculated the imaginary component of coherence (IC) (Nolte, et al., 2004) between each voxel and its homologous contralateral region during the entire auditory stimulus, hence resulting in a measure of interhemispheric coherence or interhemispheric oscillation synchrony. We calculated the spectrum of interhemispheric coherence between 1 and $20 \mathrm{~Hz}$ in the Heschl Gyrus by averaging across its voxels. Differences between rest, MBs and BBs conditions were specifically tested in alpha and theta band given the auditory stimulation that we used, using a two-tailed paired $t$-test.

Finally, to ensure not to miss coherence changes in other brain areas, we created an interhemispheric FC map of the whole brain in a voxel-wise manner. Differences between rest, $\mathrm{MBs}$ and $\mathrm{BBs}$ conditions were tested with statistical non-parametric mapping for the voxel-wise map.

\subsection{Experiment 2}

We created two sets of a dichotic digit task to assess if BBs could increase binaural auditory discriminability. Each set contained 20 trials, composed of numbers ranging from 1 to 12 spoken by a male native French speaker. Four different numbers were simultaneously presented to both ears, two per ear, and the participants were instructed to recognize the four different numbers of each trial. One set of the test was presented before and after having 
listened to three minutes of a $10 \mathrm{~Hz}$ BBs ( $395 \mathrm{~Hz}$ and $405 \mathrm{~Hz}$ tones) and the other set before and after the corresponding MBs. Sounds and tests were presented in counterbalanced order. Eighteen healthy subjects participated (10 females, mean age of 26 years ranging from 21 to 32 years old). Performance was quantified by calculating the number of correct answers out of the 80 total heard numbers (20 trials with 4 digits each). Improvement was computed for each condition by subtracting the number of correct answers after the auditory stimulation from the number of correct answers before stimulation. Differences in improvement between BBs and MBs conditions were tested for significance with a two-tailed paired $t$-test. We then confirm null effects using JZS Bayes factor tests with default prior scales (Morey \& Rouder, 2011) so that a Bayes factor $(\mathrm{B})<0.33$ implies substantial evidence for the null hypothesis. Analyses were performed with R (R Development Core Team, 2013) including the BayesFactor package (Morey \& Rouder, 2015).

\section{Results}

\subsection{Experiment 1}

No significant differences were found in oscillation power in the auditory cortices between BBs, MBs and resting state conditions during $4 \mathrm{~Hz}$ and $10 \mathrm{~Hz}$ beats (minimal voxel value in 911Hz band: $p>0.26$ and $p>0.39$ respectively)

Conversely, interhemispheric coherence between the Heschl gyri was significantly greater in the alpha band $(9-11 \mathrm{~Hz})$ during $10 \mathrm{~Hz}$ BBs stimulation compared to a $10 \mathrm{~Hz} \mathrm{MB}$ condition $\left(\mathrm{t}_{8}=3.24 ; \mathrm{p}=0.012\right)$ (Figure $\left.1 \mathrm{~A}\right)$ and resting state $\left(\mathrm{t}_{8}=3.83 ; \mathrm{p}=.005\right)$. A similar coherence spectrogram pattern was found during $4 \mathrm{~Hz}$ BBs stimulation compared to $4 \mathrm{~Hz} \mathrm{MB}$ (Figure $1 \mathrm{~A}$ $\&$ Figure 1B), with a significant increase in interhemispheric coherence at $9 \mathrm{~Hz}\left(\mathrm{t}_{4}=3.87\right.$; 
$\mathrm{p}=0.018$ ) and no significant difference observed around $4 \mathrm{~Hz}$ (minimal $\mathrm{p}$ value found at $3 \mathrm{~Hz}=$ 0.17) (Figure 1B).

A voxel-wise analysis of interhemispheric coherence showed that this increase during the BBs condition was relatively specific to the primary auditory cortex, although changes were also observed in frontal and occipital areas (Figure 1C).

\subsection{Experiment 2}

We observed an improvement in the dichotic digit test in both conditions ( $B B s t_{17}=5.55$, $\mathrm{p}<.001 ; \mathrm{MBs}_{17}=2.64, \mathrm{p}=.02$ ) but no statistically significant difference between conditions $\left(\mathrm{t}_{17}=.268 ; \mathrm{p}=.79\right)$ (Figure 2). Bayes factor confirmed the null effect showing that Ho was around 5 times more likely then the alternative hypothesis $(B=0.18)$. Complementary statistical analysis revealed that the performance achieved before the auditory stimulus (i.e. baseline score) was negatively correlated to the improvement after exposition to BBs ( $\mathrm{r}=-$ $.576 ; \mathrm{p}=.01)$. Conversely no similar correlation was observed with improvement under MBs (r $=-.11 ; \mathrm{p}=.65)$.

\section{Discussion}

In this study we found that, compared to $\mathrm{MBs}$ and resting state, BBs increased interhemispheric coherence between the auditory cortices. Moreover, both $10 \mathrm{~Hz}$ and $4 \mathrm{~Hz}$ beats increased coherence selectively in the alpha band. These changes in oscillation synchrony were not associated with changes in amplitude. Despite these electrophysiological findings, no corresponding behavioral effect could be demonstrated after the stimulation. 
Our study provides the first evidence for the long suspected increased interhemispheric synchrony under BBs and insights into underlying mechanisms. Our observation is comparable to previous reports that cognitive tasks can modify alpha-band coherence. For example, increased alpha oscillation synchrony has been reported during object recognition (Mima, et al., 2001), spatial attention (Doesburg, et al., 2009), error processing (van Driel, et al., 2012), mental calculation (Palva, et al., 2005) or memory tasks (Haegens, et al., 2010). We consider BBs as a challenging listening situation with a binaural perceptual conflict that the auditory system solves by increasing communication between both auditory cortices. This in turns leads to an increase in phase locked synchrony among neurons oscillating in alpha band. Hence, we surmise that the increase in the alpha-band coherence observed here reflects binaural integration.

One might be surprised by the selective effect in alpha frequencies only. Neurons preferably oscillate at certain frequencies that are typically biologically important (Herrmann, 2001). As for several other sensory modalities, alpha oscillations are the predominant frequency in the auditory cortex and can be modulated by auditory stimuli (Hartmann, et al., 2012; Strauß, et al., 2014). We postulate that BBs effect on interhemispheric communications is restricted to the alpha band because it is the main spontaneous oscillation band and is involved in most of the interactions between cortical areas (von Stein, et al., 2000).

Conversely, we didn't find an increase in power during $10 \mathrm{~Hz}$ and $4 \mathrm{~Hz}$ BBs compared to rest or the corresponding MBs. The absence of alpha power modulation during $10 \mathrm{~Hz} \mathrm{BBs}$ is consistent with previous studies (Goodin, et al. 2012; Vernon, et al., 2014). Regarding the lack of modulation of theta power during $4 \mathrm{~Hz}$ BBs, both similar (Wahbeh, et al., 2007) and opposite results (Brady \& Stevens, 2000; Karino, et al., 2006; Pratt, et al., 2010) have been 
reported in the literature. These studies have different designs in terms of recording device (EEG vs MEG) or exposition time (from $2 \mathrm{~s}$ up to 20 minutes) that could account for these discrepancies. In any case, consistent with our findings, increase in alpha oscillation synchrony has already been reported several times without corresponding modification of oscillation power (Mima, et al., 2001; Vanni, et al., 1997).

Some authors have hypothesized that EEG changes observed under BBs are elicited through an entertainment effect (Vernon, 2009), but our findings cannot be explained by this mechanism. We found that BBs stimulation with $4 \mathrm{~Hz}$ beats increases interhemispheric coherence in non-harmonic alpha frequencies, which is difficult to bring in line with entrainment. Moreover, in our study coherence increased independently of power changes whereas synchronization of neuron's firing to the frequency of the beating stimulus would theoretically lead to an amplitude increase of the corresponding EEG frequency. On the basis of these findings, we assume that the increase in the interhemispheric coherence observed here could not be explained by synchronization to the external rhythmic force.

Given the observed electrophysiological effect, we tried to improve binaural auditory discrimination abilities using BBs in healthy subject. However, no significant effect was observed compared to a monaural control condition. One possible explanation for this finding is that our behavioral task was not sufficiently sensitive to reveal an improvement in auditory function. However, dichotic digit tests have been successfully used to assess interhemispheric integration (Musiek, 1983; Bellis \& Wilber, 2001; Westerhausen \& Hugdahl, 2008). A more likely explanation is that the increase in interhemispheric collaboration was limited to the simulation period and not associated with a corresponding aftereffect at the time at which we performed our dichotic digit task. It would have been interesting to record EEG not only 
during, but also after auditory stimulation in order to confirm the lack of aftereffect also on the neural level.

Our study does not exclude that repetitive BBs stimulation over several days would eventually result in measurable aftereffects. However, there is currently no evidence for this possibility. Finally, we found that the less the subjects were able to distinguish binaurally presented numbers at baseline, the more BBs increased their performance. Since no such correlation was found for MBs, we might speculate that BBs could be more beneficial for patients with impairment in interhemispheric interaction. This is consistent with previous observations on BBs behavioral effect. For instance, Brady and Steven found that hypnotic susceptibility increased only among low and medium-susceptible subjects (Brady \& Stevens, 2000). However, this will need to be confirmed by more robust evidence.

In conclusion, this study demonstrates for the first time a modulation of interhemispheric coherence by BBs. However, a single session of BBs does not seem to result in corresponding behavioral aftereffects of improved interhemispheric communication.

\section{Acknowledgments}

Authors would like to thank Prof. A. Schnider for precious comments about the manuscript

\section{References}

Aertsen,A.M., Gerstein,G.L., Habib,M.K., Palm,G. 1989. «Dynamics of Neuronal Firing Correlation: Modulation of "Effective Connectivity". » Journal of Neurophysiology 61 (5): $900-917$. 
Atwater,F.H., 1997. «Accessing anomalous states of consciousness with a binaural beat technology», Journal of Scientific Exploration, Volume 11, Issue 3, Pages 263-274

Atwater,F.H. 2004. «The Hemi-Sync® Process ». VA: The Monroe Institute.

Becher,A., Höhne,M., Axmacher,N., Chaieb,L., Elger,C.E., Fell,J. 2014. « Intracranial Electroencephalography Power and Phase Synchronization Changes during Monaural and Binaural Beat Stimulation ». European Journal of Neuroscience, octobre, n/a - n/a. doi:10.1111/ejn.12760.

Bellis,T.J., Wilber,L. A. 2001. «Effects of Aging and Gender on Interhemispheric Function ». Journal of Speech, Language, and Hearing Research: JSLHR 44 (2): 246- 63.

Brady,B., Stevens,L.. 2000. «Binaural-Beat Induced Theta EEG Activity and Hypnotic Susceptibility ». The American Journal of Clinical Hypnosis 43 (1): 53 - 69.

Dalal,S.S., Zumer,J.M., Guggisberg,A.G., Trumpis,M., Wong,D.D.E., Sekihara,K., Nagarajan,S.S., 2011. MEG/EEG source reconstruction, statistical evaluation, and visualization with NUTMEG. Comput. Intell. Neurosci. 2011, 758973. doi:10.1155/2011/758973

Doesburg,S.M., Green, J.J., McDonald, J. J., Ward,L.M., 2009. « From Local Inhibition to Long-Range Integration: A Functional Dissociation of Alpha-Band Synchronization across Cortical Scales in Visuospatial Attention ». Brain Research 1303 (décembre): 97- 110. doi:10.1016/j.brainres.2009.09.069.

Draganova,R., Ross,B. Wollbrink, A., Pantev,C., 2008. «Cortical Steady-State Responses to Central and Peripheral Auditory Beats ». Cerebral Cortex (New York, N.Y.: 1991) 18 (5): 1193- 1200. doi:10.1093/cercor/bhm153. 
Engel,A.K., König,P., Kreiter,A.K., Schillen,T.B., Singer,W., 1992. « Temporal coding in the visual cortex: new vistas on integration in the nervous system ». Trends in Neurosciences 15 (6): 218- 26. doi:10.1016/0166-2236(92)90039-B.

Fox,M. D., Snyder,A.Z., Vincent,J.L., Raichle,M.E. 2007. «Intrinsic Fluctuations within Cortical Systems Account for Intertrial Variability in Human Behavior ». Neuron 56 (1): 171 - 84. doi:10.1016/j.neuron.2007.08.023.

Fries,P. 2005. «A Mechanism for Cognitive Dynamics: Neuronal Communication through Neuronal Coherence ». Trends in Cognitive Sciences 9 (10): 474- 80. doi:10.1016/j.tics.2005.08.011.

Gao,X., Cao,H., Ming,D., Qi,H., Wang,X., Wang,X., Chen,R., Zhou,P. 2014. « Analysis of EEG Activity in Response to Binaural Beats with Different Frequencies ». International Journal of Psychophysiology: Official Journal of the International Organization of Psychophysiology 94 (3): 399- 406. doi:10.1016/j.ijpsycho.2014.10.010.

Goodin,P., Ciorciari,J., Baker,K., Carey,A.M., Harper,M., Kaufman.J., 2012. « A HighDensity EEG Investigation into Steady State Binaural Beat Stimulation ». PloS One 7 (4): e34789. doi:10.1371/journal.pone.0034789.

Gray,C. M., Singer,W.. 1989. «Stimulus-Specific Neuronal Oscillations in Orientation Columns of Cat Visual Cortex ». Proceedings of the National Academy of Sciences 86 (5): $1698-1702$.

Grose,J.H., Mamo,S.K., 2012. «Electrophysiological Measurement of Binaural Beats: Effects of Primary Tone Frequency and Observer Age ». Ear and Hearing 33 (2): 187- 94. doi:10.1097/AUD.0b013e318230bbbd. 
Guggisberg, A.G., Honma,S.M., Findlay,A.M., Dalal,S.S., Kirsch,H.E., Berger,M.S., Nagarajan,S.S., 2008. Mapping functional connectivity in patients with brain lesions. Ann. Neurol. 63, 193-203.

Guggisberg,A.G, Dalal,SS, Zumer,JM, Wong,DD, Dubovik,S, Michel,CM, Schnider,A., Localization of cortico-peripheral coherence with electroencephalography. Neuroimage 2011;57:1348-57.

Guggisberg,A.G., Rizk,S., Ptak,R., Di Pietro,M., Saj,A., LazeyrasF.,, Lovblad,KO., Schnider,A., et Pignat,J.M.. 2015. « Two Intrinsic Coupling Types for Resting-State Integration in the Human Brain ». Brain Topography 28 (2): 318- 29. doi:10.1007/s 10548-014-0394-2.

Haegens,S., Osipova,D., Oostenveld,R., Jensen,O., « Somatosensory working memory performance in humans depends on both engagement and disengagement of regions in a distributed network.». Hum Brain Mapp. 2010 Jan;31(1):26-35. doi: 10.1002/hbm.20842.

Hartmann,T., Schlee,W., Weisz,N., 2012. «It’s Only in Your Head: Expectancy of Aversive Auditory Stimulation Modulates Stimulus-Induced Auditory Cortical Alpha Desynchronization ». NeuroImage 60 (1): 170- 78. doi:10.1016/j.neuroimage.2011.12.034.

Herrmann,C.S. 2001. «Human EEG Responses to 1-100 Hz Flicker: Resonance Phenomena in Visual Cortex and Their Potential Correlation to Cognitive Phenomena ». Experimental Brain Research 137 (3-4): 346- 53.

Hummel,F., Gerloff,C., 2005. «Larger Interregional Synchrony Is Associated with Greater Behavioral Success in a Complex Sensory Integration Task in Humans ». Cerebral Cortex 15 (5): 670- 78. doi:10.1093/cercor/bhh170. 
Karino,S., Yumoto,M., Itoh,K., Uno,A., Matsuda,M., Yamakawa,K., Sekimoto,M., Kaneko,Y., Kaga,K., 2004. Magnetoencephalographic study of human auditory steadystate responses to binaural beat. International Congress Series 1270, 169-172.

Karino,S., Yumoto,M., Itoh,K., Uno,A., Yamakawa,K., Sekimoto,S., Kaga,K., 2006. « Neuromagnetic Responses to Binaural Beat in Human Cerebral Cortex ». Journal of Neurophysiology 96 (4): 1927- 38. doi:10.1152/jn.00859.2005.

Kuwada,S., Yin,T.C., Wickesberg,R.E., 1979. « Response of Cat Inferior Colliculus Neurons to Binaural Beat Stimuli: Possible Mechanisms for Sound Localization ». Science (New York, N.Y.) 206 (4418): 586- 88.

Lane,J.D., Kasian,S.J., Owens,J.E., Marsh,G.R., 1998. «Binaural Auditory Beats Affect Vigilance Performance and Mood ». Physiology \& Behavior 63 (2): 249- 52.

Le Scouarnec,R.P., Poirier,R.M., Owens,J.E., Gauthier,J., Taylor,A.G., Foresman,P.A., 2001. «Use of Binaural Beat Tapes for Treatment of Anxiety: A Pilot Study of Tape Preference and Outcomes ». Alternative Therapies in Health and Medicine 7 (1): 58 - 63.

Mima,T., Oluwatimilehin,T., Hiraoka,T., Hallett,M., 2001. «Transient Interhemispheric Neuronal Synchrony Correlates with Object Recognition ». The Journal of Neuroscience: The Official Journal of the Society for Neuroscience 21 (11): 3942- 48.

Moore,B.C.J., 2012. An Introduction to the Psychology of Hearing. BRILL.

Morey,R.D., Rouder,J.N., 2011. Bayes factor approaches for testing interval null hypotheses. Psychol. Methods 16, 406-419. doi:10.1037/a0024377

Musiek,F.E., 1983. «Assessment of Central Auditory Dysfunction: The Dichotic Digit Test Revisited ». Ear and Hearing 4 (2): $79-83$.

Nolte,G., Bai,O., Wheaton.L,, Mari,Z., Vorbach,S., Hallett,M., 2004. « Identifying True Brain Interaction from EEG Data Using the Imaginary Part of Coherency ». Clinical 
Neurophysiology: Official Journal of the International Federation of Clinical

Neurophysiology 115 (10): 2292- 2307. doi:10.1016/j.clinph.2004.04.029.

Oster,G., 1973. «Auditory Beats in the Brain ». Scientific American 229 (4): 94- 102.

Padmanabhan,R., Hildreth,A.J., Laws,D., 2005. « A Prospective, Randomised, Controlled

Study Examining Binaural Beat Audio and Pre-Operative Anxiety in Patients Undergoing

General Anaesthesia for Day Case Surgery ». Anaesthesia 60 (9): $874-77$.

doi:10.1111/j.1365-2044.2005.04287.x.

Palva,J.M., Palva,S., Kaila,K., 2005. «Phase Synchrony among Neuronal Oscillations in the Human Cortex ». The Journal of Neuroscience: The Official Journal of the Society for Neuroscience 25 (15): 3962- 72. doi:10.1523/JNEUROSCI.4250-04.2005.

Pratt,H., Starr,A., Michalewski,HJ., Dimitrijevic,A., Bleich,N., Mittelman,N., 2009. « Cortical Evoked Potentials to an Auditory Illusion: Binaural Beats ». Clinical Neurophysiology: Official Journal of the International Federation of Clinical Neurophysiology 120 (8): 1514- 24. doi:10.1016/j.clinph.2009.06.014.

Pratt,H., Starr,A., Michalewski,HJ., Dimitrijevic,A., Bleich,N., Mittelman,N., 2010. «A Comparison of Auditory Evoked Potentials to Acoustic Beats and to Binaural Beats ». Hearing Research 262 (1-2): 34- 44. doi:10.1016/j.heares.2010.01.013.

Reedijk,S.A., Bolders,A., Hommel,B., 2013. « The Impact of Binaural Beats on Creativity ». Frontiers in Human Neuroscience 7: 786. doi:10.3389/fnhum.2013.00786.

Rhodes,L. 1993. «Use of the Hemi-Sync super sleep tape with a preschool-aged child». Hemi-Sync Journal, XI(4), pp. iv-v.

Schwarz,D.W.F., Taylor,P., 2005. « Human auditory steady state responses to binaural and monaural beats ». Clinical Neurophysiology 116 (3): 658- 68. doi:10.1016/j.clinph.2004.09.014. 
Sekihara,K,. Nagarajan,S.S., Poeppel,D., Marantz,A., 2004. «Asymptotic SNR of Scalar and Vector Minimum-Variance Beamformers for Neuromagnetic Source Reconstruction ». IEEE Transactions on Bio-Medical Engineering 51 (10): 1726- 34. doi:10.1109/TBME.2004.827926.

Spinelli,L., Andino,S.G., Lantz,G., Seeck,M., Michel,C.M., 2000. «Electromagnetic Inverse Solutions in Anatomically Constrained Spherical Head Models ». Brain Topography 13 (2): $115-25$.

Strauß,A., Wöstmann,M., Obleser,J., 2014. «Cortical alpha oscillations as a tool for auditory selective inhibition ». Frontiers in Human Neuroscience 8 (mai). doi:10.3389/fnhum.2014.00350.

Tzourio-Mazoyer,N., Landeau,B., Papathanassiou,D., Crivello,F. Etard,O., Delcroix,N., Mazoyer,B., Joliot,M., 2002. «Automated Anatomical Labeling of Activations in SPM Using a Macroscopic Anatomical Parcellation of the MNI MRI Single-Subject Brain ». NeuroImage 15 (1): 273- 89. doi:10.1006/nimg.2001.0978.

van Driel,J., Ridderinkhof,K.R., Cohen,M.X., 2012. « Not All Errors Are Alike: Theta and Alpha EEG Dynamics Relate to Differences in Error-Processing Dynamics ». The Journal of Neuroscience: The Official Journal of the Society for Neuroscience 32 (47): 16795- 806. doi:10.1523/JNEUROSCI.0802-12.2012.

Vanni,S., Revonsuo,A., Hari,R., 1997. «Modulation of the Parieto-Occipital Alpha Rhythm during Object Detection ». The Journal of Neuroscience 17 (18): 7141 - 47.

Varela,F., Lachaux,J.P., Rodriguez,E., Martinerie,J., 2001. «The Brainweb: Phase Synchronization and Large-Scale Integration ». Nature Reviews Neuroscience 2 (4): 229- 39. doi:10.1038/35067550. 
Vernon,D., 2009. In Human Potential: Exploring Techniques Used to Enhance Human Performance., Routledge. London.

Vernon,D., Peryer,G., Louch,J., Shaw,M., 2014. « Tracking EEG Changes in Response to Alpha and Beta Binaural Beats ». International Journal of Psychophysiology: Official Journal of the International Organization of Psychophysiology 93 (1): 134- 39. doi:10.1016/j.ijpsycho.2012.10.008.

von Stein,A., Chiang,C., König,P., 2000. « Top-down processing mediated by interareal synchronization ». Proceedings of the National Academy of Sciences of the United States of America 97 (26): 14748- 53.

Wahbeh,H., Calabrese,C., Zwickey,H., Zajdel,D., 2007. «Binaural Beat Technology in Humans: A Pilot Study to Assess Neuropsychologic, Physiologic, and Electroencephalographic Effects ». Journal of Alternative and Complementary Medicine (New York, N.Y.) 13 (2): 199- 206. doi:10.1089/acm.2006.6201.

Wernick,J.S., Starr,A., 1968. « Binaural Interaction in the Superior Olivary Complex of the Cat: An Analysis of Field Potentials Evoked by Binaural-Beat Stimuli. » Journal of Neurophysiology 31 (3): 428- 41.

Westerhausen,R., Hugdahl,K., 2008. « The Corpus Callosum in Dichotic Listening Studies of Hemispheric Asymmetry: A Review of Clinical and Experimental Evidence ». Neuroscience and Biobehavioral Reviews 32 (5): 1044- 54. doi:10.1016/j.neubiorev.2008.04.005.

Wilson,E.S., 1990. « Preliminary study of the Hemi-Sync sleep processor ». Colorado Association for Psychophysiologic Research. 


\section{Legends to Tables and Figures}

Figure 1: Effect of BBs on interhemispheric coherence

Both (A) $10 \mathrm{~Hz}$ BBs and (B) $4 \mathrm{~Hz}$ BBs produced a significant increase in interhemispheric coherence between the primary auditory cortices compared to MBs in alpha frequencies (911Hz). Note the similarity of the patterns of the two graphs. (C) Subtraction of FC map during $\mathrm{BBs}$ from the $\mathrm{FC}$ map during MBs shows a significant increase in interhemispheric alpha-band coherence in fronto-temporo-parietal areas around the auditory cortex $(\mathrm{p}<0.05$ uncorrected). 
Figure 2: Behavioral performance in dichotic digit test.

Subjects improved after both stimulation conditions, but no statistically significant difference between BBs and MBs was found $(\mathrm{p}=.79)$ 

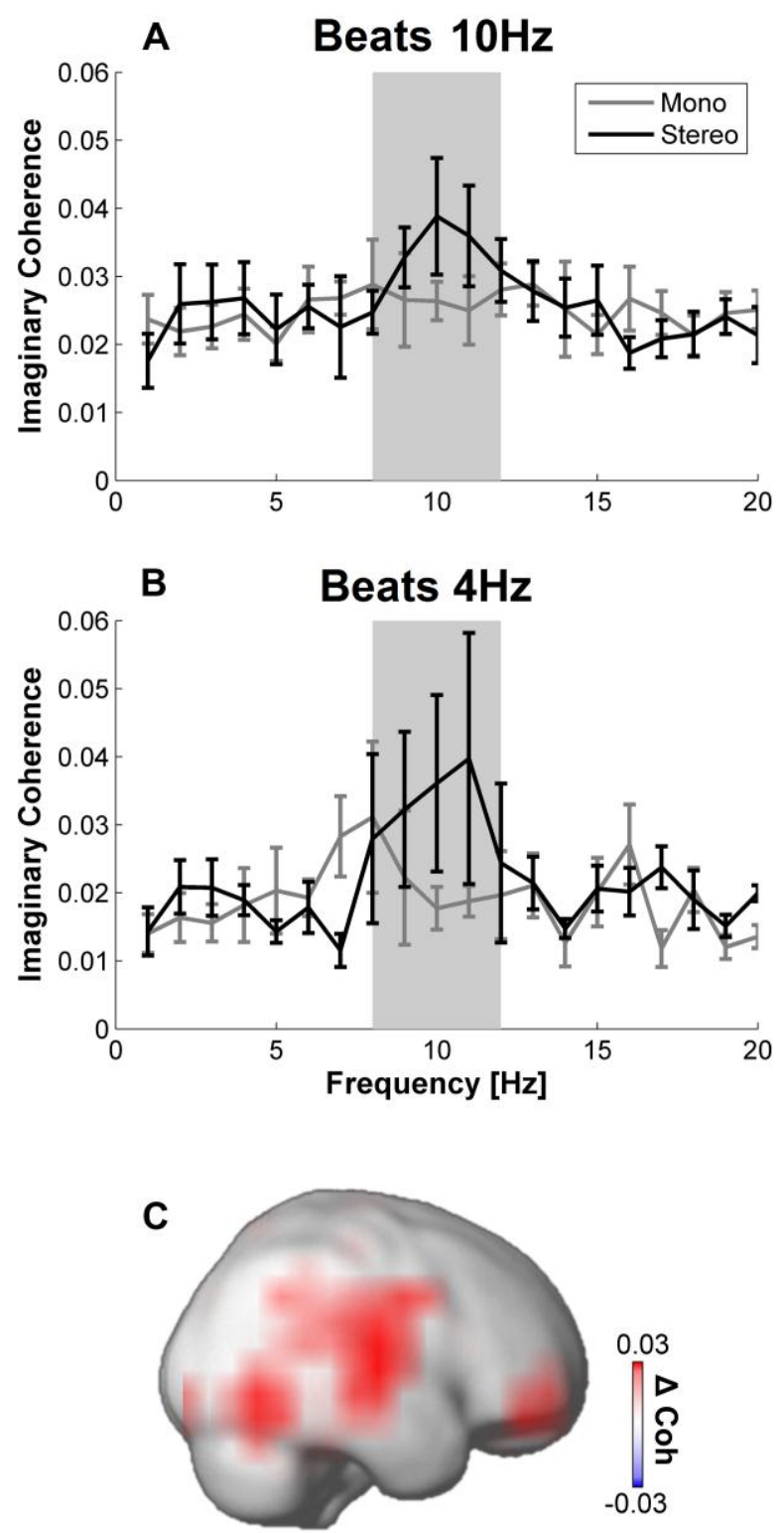


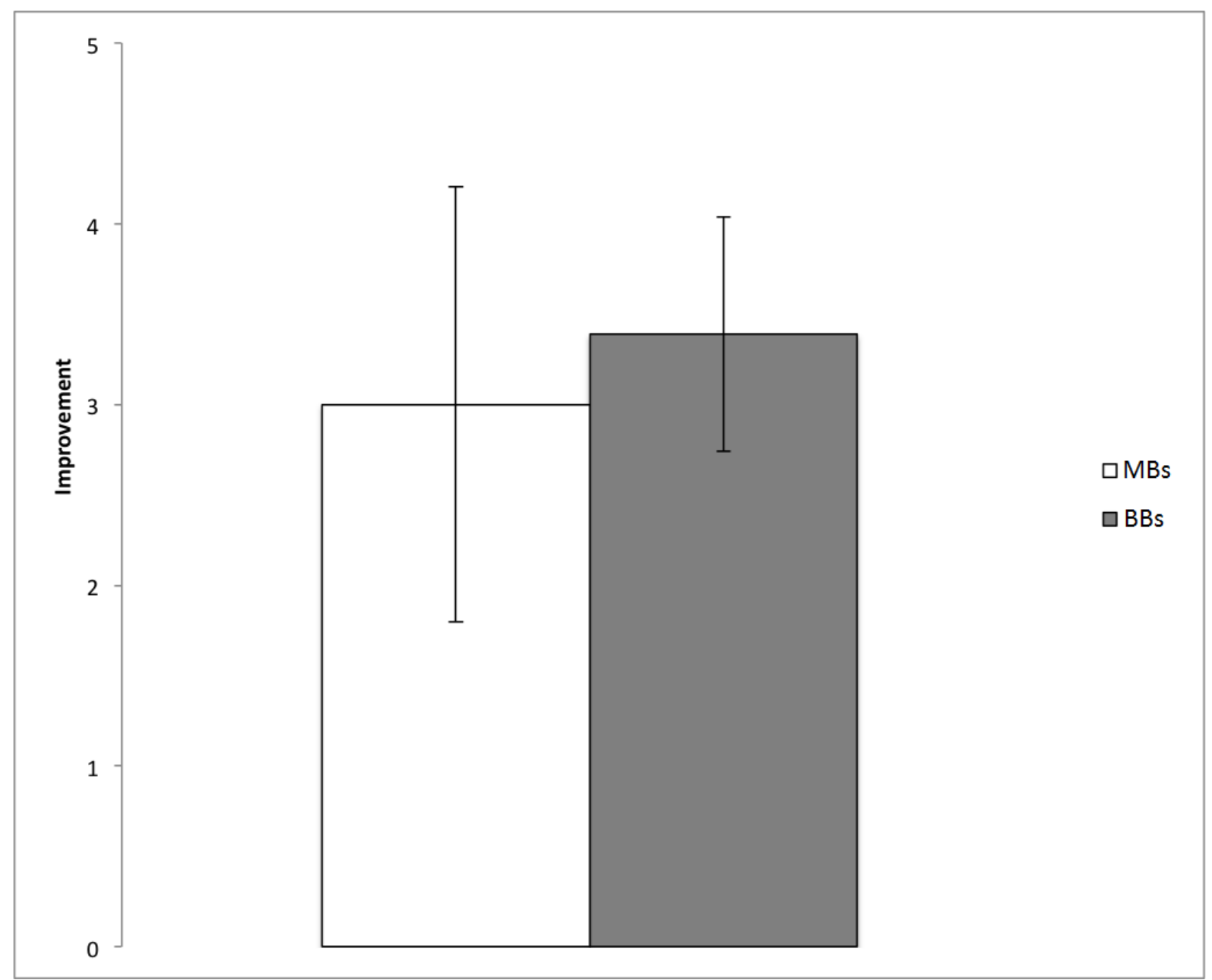

